\title{
Apparent distance between profiles of faces with dynamic properties that represent interpersonal relationships
}

\author{
HIROKO OKADA and SEYMOUR WAPNER \\ Clark University, Worcester, Massachusetts
}

\begin{abstract}
Sixty-four subjects ( 32 male and 32 female) adjusted two luminous profiles of faces in a dark room, to a standard distance of $12 \mathrm{in}$. $(30 \mathrm{~cm})$ between them. The profile pairs were systematically varied in four ways: (1) facing each other and "smiling"; (2) facing each other and "angry"; (3) back to back and "smiling"; and (4) back to back and "angry." Orientation and emotion had significant effects on adjustment to the standard distance between profiles: mean adjustment was smaller for face-to-face than for back-to-back orientation, and adjustment was smaller for representations with positive, as opposed to negative, emotion. Thus, evidence is available that directional dynamics in pictorial (face-profile) representations of interpersonal relationships have significant effects on perceived distance.
\end{abstract}

This study represents an attempt to link some features of perception with some aspects of pictorial representations (profiles of faces) of interpersonal relationships. It involves Werner's $(1940,1956)$ notion of physiognomic perception, a term that refers to the dynamic, expressive, vectorial qualities of percepts-such as a bird in flight, a line as cruel or happy, or colors as strong or weakwhich are to be distinguished from geometrical-technical matter-of-fact qualities-such as shape, size, and location.

The existence of directional dynamics in pictorial representations has been experimentally demonstrated with respect to a variety of situations. For example, Werner and Wapner (1954) provided evidence that left-right directional dynamics - triangles, faces, birds/planes pointing left and right-significantly affect spatial localization, as measured by the position of the apparent median plane (straight ahead), which shifts opposite the direction of the dynamics. Parallel findings have been obtained for the up-down dimension of space (Kaden, Wapner, \& Werner, 1955), with real and apparent motion (Comalli, 1960; Comalli, Werner, \& Wapner, 1957; Miller, Werner, \& Wapner, 1958) and with perceived size and distance (Lindauer, 1984, 1986, 1988; Schlesinger, 1980).

Assuming that profile representations of interpersonal relations have dynamics (e.g., profiles of people facing each other vs. back-to-back, a smiling vs. an angry man and woman looking at each other), they should differentially affect perceived left-right distance between the profiles. If both profiles have a positive emotional quality (e.g., both are smiling), they can be expected to have a dynamic, expressive quality of approach, with the opposite effect if both are expressing anger or frowning (cf.

Correspondence should be addressed to Seymour Wapner, Frances L. Hiatt School of Psychology, Clark University, 950 Main Street, Worcester, MA 01610-1477.
deRivera, 1977). In the former, when subjects are requested to place the profiles at a given fixed distance, to compensate for the directional dynamics toward each of the faces constituting a pair, it can be expected that the profiles would be placed farther apart than if the opposite dynamics (away from each other) existed.

\section{METHOD}

\section{Subjects}

The subjects were 64 ( 32 men and 32 women) volunteers between the ages of 19 and 35 .

Task

The task for the subject was-after viewing a pair of luminous profiles that depicted heads of people located in a horizontal plane, initially close (12 in.) or initially far apart (20 in.)-to tell the experimenter how to adjust the two dimly lit profiles so that they appeared to be 12 in. $(30 \mathrm{~cm})$ apart.

\section{Apparatus}

A chair with a headrest was located $2 \mathrm{~m}$ in front of an apparatus that contained the two dimly lit profiles, which could be moved horizontally. The two profiles were each cut from a $20 \times 20$ in. sheet of black cardboard construction paper; the cardboard that remained was attached to the translucent front face of a lightbox. The two lightboxes each contained a $5-\mathrm{W}$ bulb operating at $30 \mathrm{~V}$, which made the profiles luminous when the room was dark. The boxes were placed on a track in the frontoparallel plane $2 \mathrm{~m}$ from the subject. The boxes were simultaneously movable to make the distance between the profiles smaller or larger. The subject's chair was located so that its objective median plane was exactly at the midpoint between the two profiles.

\section{Stimuli}

Four profiles were used. They portrayed a man and woman facing each other and smiling; a man and woman facing each other and angry; a man and woman back to back and smiling; and a man and woman back to back and angry. There were 16 test trials, involving all combinations of two levels for each of four factors: (1) orientation-facing each other versus back to back; (2) emotional expression-positive versus negative expression; (3) position with respect to the median planeman on left side versus man on right side; (4) starting distance of 
stimuli- 4 in. versus 20 in. In combination, this made for a total of 16 test conditions on which each subject was tested.

\section{Design}

The 16 test conditions were arranged as a Latin square, with 4 men and 4 women in each of the 16 sequences.

\section{Procedure}

The subject was led into the darkened room while blindfolded and was then seated in the chair, with the headrest adjusted so that the subject was comfortable but unable to move during the test procedure. The subject was asked to remove the blindfold and open his or her eyes; the subject was then asked, "Is there anything particular about these profiles? What is characteristic of them?'” Next, the subject was asked how the two profiles should be adjusted to make them appear 12 in. apart: "Should they be moved closer, or farther away?" After the subject gave his or her instructions to the experimenter, the two boxes were moved simultaneously in small steps until the subject was satisfied that the distance between profiles appeared to be 12 in. After the subject had completed a given trial, the subject was told to put the blindfold back on again and close his or her eyes. This permitted the experimenter to measure the distance between the profiles while the subject could not see the room, the apparatus, or what the experimenter was doing. After making the measurement, the experimenter set up the combination of profiles for the next trial and proceeded in the same way until the 16 trials were completed.

\section{Statistical Analysis}

The data were analyzed as independent groups (gender) with a repeated measures (orientation, emotional expression, starting distance, position) analysis of variance.

\section{RESULTS}

Before presentation of the main findings, it should be noted that all subjects' descriptions of the profiles indicated that they attributed positive (e.g., smiling, happy, laughing) or negative (e.g., frowning, unhappy, sad, disgusted) emotion to them in keeping with the intended representation.

The overall effects of the main factors of orientation of profiles constituting a pair and emotion expressed were significant. More specifically, the overall mean adjusted distance between profiles facing each other was $22.10 \mathrm{~cm}$, whereas the mean for profiles back to back was $22.79 \mathrm{~cm}$ $[F(1,62)=27.71, p<.01]$. Of the 64 subjects, $77 \%$ (49) showed greater distance scores, whereas only $23 \%$ (15) showed shorter distance scores, for profiles oriented back to back ( $p<.01$, sign test).

The overall adjusted mean distance for profiles with negative emotion was $22.61 \mathrm{~cm}$, whereas the mean was $22.29 \mathrm{~cm}$ for profiles with positive emotion $[F(1,61)=$ $10.87, p<.01$ ]. Of the 64 subjects, $70 \%$ (45) showed greater distance scores whereas only $30 \%$ (45) showed shorter distance scores for profiles with negative emotional expression $(p<.01$, sign test).

It should be noted that, although the factors of both orientation and emotion were significant, the effects were the opposite of what was predicted, given the assumption that the distance adjusted would be compensatory to the directional dynamic features of the stimuli constituting a pair. The order of means from the shortest to the longest distance between profiles constituting a pair was as follows: facing with positive emotion $(M=22.01 \mathrm{~cm})$; fac- ing with negative emotion $(M=22.20 \mathrm{~cm})$; back to back with positive emotion $(M=22.57 \mathrm{~cm})$; and back to back with negative emotion $(M=23.01 \mathrm{~cm})$.

In keeping with many investigations of space perception in which psychophysical methods of adjusting stimuli to some standard have been used, the initial starting distance between the profiles had a significant effect on the final adjustment (cf. Werner \& Wapner, 1952). The overall mean for the large $(50-\mathrm{cm})$ starting distance was significantly greater $(M=23.65 \mathrm{~cm})$ than the mean for the smaller $(10-\mathrm{cm})$ starting distance $[F(1,62)=67.92$, $p<.01]$.

\section{DISCUSSION}

In the present experiment, we obtained evidence in keeping with the general hypothesis that directional dynamics in profiles constituting a pair make for significant differences in the apparent distance between the two profiles, depending on (1) whether the profiles are facing each other or are back to back and (2) whether the profiles are pictured with positive or with negative emotion. As noted above, although these general hypotheses were confirmed, the specific directional effects of orientation and emotion represented were opposite those predicted. The mean adjustment between profiles constituting a pair was not larger when dynamics were directed toward each other (compensatory relationship); instead, it was smaller.

These directional findings are puzzling, although it is true that parallel specific findings were obtained in another study of apparent distance between stimuli. This study (Teft, Wapner, Werner, \& McFarland, 1964), in which the relationship between numerical and perceived extent was examined, showed that if an extent is demarcated by two numbers (between 1 and 9), a visual extent demarcated by a smaller numerical interval (e.g., 1-2) appears equal to a larger numerical interval (e.g., 2-9) when the former is physically smaller than the latter. As in the present study, the finding was the opposite of what was expected. Instead of adjustment of the stimuli to a given standard operating in a compensatory manner, there was a correspondence between the magnitude of numerical (conceptual) and perceived distance. Teft et al. (1964) offered the explanation that for subjects to achieve visual equivalence, the two physical extents compared had to be in correspondence with the relationship of the cognitively apprehended numerical intervals.

In parallel fashion, in the present experiment, the adjustment of the profiles to a required standard extent is also in keeping with the correspondence principle: That is, with dynamic vectorial properties in a configuration operating to decrease extent (dynamics inward-face-toface profiles, with positive emotion), there is a correspondent decrease in adjustment to a standard; with dynamic properties operating to increase an extent (dynamics outward-back-to-back profiles, with negative emotion) there is a correspondent increase in adjustment to a standard. Further experimentation will be required for the assessment of the generality of this correspondent relation between dynamics and adjustment to a standard distance.

Taken as a whole, the findings reported here have significance insofar as they demonstrate the efficacy of directional, vectorial properties in interpersonal pictorial representations varying with respect to orientation and emotion; and, in general, they demonstrate ways in which cognition is compromised by emotion.

\section{REFERENCES}

Comalli, E. E., JR. (1960). Studies in physiognomic perception: VI. Differential effects of directional dynamics of pictorial objects on real and apparent motion in artists and chemists. Journal of Psychology, 49, 99-109.

Comalli, E. E., Jr., Werner, H., \& WAPNer, S. (1957). Studies in physiognomic perception: III. Effect of directional dynamics and meaning-induced sets on autokinetic motions. Journal of Psychology, 43, 289-299. 
DeRivera, J. (1977). A structural theory of the emotions. Psychological Issues, 10(4, Monograph 40).

Kaden, S. E., WaPNer, S., \& Werner, H. (1955). Studies in physiognomic perception: II. Effect of directional dynamics of pictured objects and words on the position of the apparent horizon. Journal of Psychology, 39, 61-70.

Lindauer, M. S. (1984). Physiognomic perception. In R. J. Corsini (Ed.), Wiley encyclopedia of psychology (Vol. 3, pp. 34-35). New York: Wiley.

LiNDAUER, M. S. (1986). Perceiving, imagining, and preferring physiognomic stimuli. American Journal of Psychology, 99, 233-255.

LiNDAUER, M. S. (1988). Size and distance perception of the physiognomic stimulus "taketa." Bulletin of the Psychonomic Society, 26, 217-220.

Miller, A., Werner, H., \& W APNer, S. (1958). Studies in physiognomic perception: $\mathrm{V}$. Effect of ascending and descending gliding tones on autokinetic motion. Journal of Psychology, 46, 101-105.

SCHLESINGER, L. B. (1980). Physiognomic perception: Empirical and theoretical perspectives. Genetic Psychology Monographs, 101, 71-97.
Teft, L., Wapner, S., Werner, H., \& McFarland, J. H. (1964). Relation between perceptual and conceptual operations: Numerical distance and visual extent. British Journal of Psychology, 55, 421-427.

WERNER, H. (1940). Comparative psychology of mental development. New York: Harper.

WERNER, H. (1956). On physiognomic perception. In G. Kepes, The new landscape (pp. 280-283). Chicago: P. Theobald.

WERNER, H., \& WAPNER, S. (1952). Experiments on sensory-tonic field theory of perception: IV. Effect of initial position of a rod on apparent verticality. Journal of Experimental Psychology, 43, 68-74.

WERNER, H., \& WAPNER, S. (1954). Studies in physiognomic perception: I. Effect of configurational dynamics and meaning-induced sets on the position of the apparent median plane. Journal of Psychology, 38, 51-65.

(Manuscript received September 17, 1990.) 